\title{
Correction to: Holomorphic relative Hopf modules over the irreducible quantum flag manifolds
}

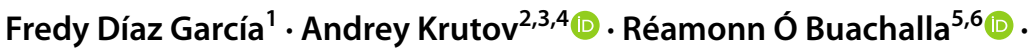 Petr Somberg ${ }^{6} \cdot$ Karen R. Strung ${ }^{3}$}

Published online: 19 February 2021

( ) The Author(s), under exclusive licence to Springer Nature B.V. 2021

\section{Correction to: Letters in Mathematical Physics https://doi.org/10.1007/s11005-020-01340-7}

The publication of this article unfortunately contained a mistake. A grant number in the acknowledgements was not correct, instead of GACR 306 - 33/1906357 it should be GA19-06357S:

Acknowledgements FDG is partially funded by Conacyt (Consejo Nacional de Ciencia y Tecnología, México). AK was supported by the QuantiXLie Centre of Excellence, a project cofinanced by the Croatian Government and European Union through the European Regional Development Fund - the

The original article can be found online at https://doi.org/10.1007/s11005-020-01340-7.

Réamonn Ó Buachalla

reamonnobuachalla@gmail.com

Fredy Díaz García

lenonndiaz@gmail.com

Andrey Krutov

krutov@math.cas.cz

Petr Somberg

somberg@karlin.mff.cuni.cz

Karen R. Strung

strung@math.cas.cz

1 Centro de Ciencias Matemáticas, Universidad Nacional Autónoma de México, Antigua Carretera a Pátzcuaro 8701 Col. Ex Hacienda San José de la Huerta, Morelia C.P. 58089, Mexico

2 Department of Mathematics, University of Zagreb, Bijenicka cesta 30, 10000 Zagreb, Croatia

3 Institute of Mathematics, Czech Academy of Sciences, Žitná 25, 11567 Prague, Czech Republic

4 Independent University of Moscow, Bolshoj Vlasyevskij Per. 11, 119002 Moscow, Russia

5 Département de Mathématiques, Faculté des sciences, Université Libre de Bruxelles, Boulevard du Triomphe, B-1050 Bruxells, Belgium

6 Mathematical Institute of Charles University, Sokolovská 83, 18675 Prague, Czech Republic 
Competitiveness and Cohesion Operational Programme (KK.01.1.1.01.0004). RÓB acknowledges FNRS support through a postdoctoral fellowship within the framework of the MIS Grant "Antipode" Grant Number F.4502.18. RÓB and PS are partially supported from the Eduard Čech Institute within the framework of the grant GAČR 19-28628X, and by the Grant GA19-06357S. Research of KRS and AK is supported by the GAČR project 20-17488Y and RVO: 67985840.

Publisher's Note Springer Nature remains neutral with regard to jurisdictional claims in published maps and institutional affiliations. 